Speculation on Primary Commodities:

The Effects of Restricted Entry

John McLaren, Columbia University

September 1992

Discussion Paper Series No. 622 


\title{
Speculation on Primary Commodities: The Effects of Restricted Entry
}

\author{
John McLaren \\ Department of Economics, \\ Columbia University
}

September, 1992

The author would like to thank Timothy Besley, Gregory Chow, Angus Deaton, Avinash Dixit, Kenneth Judd, and David Newbery as well as the Princeton Empirical Microeconomics Group for helpful comments, but accepts sole responsibility for errors. Financial support from the International Finance Section at Princeton University and from the John M. Olin Program for the Study of Economic Organization and Public Policy at Princeton is gratefully acknowledged. 


\begin{abstract}
There are doubts about the competitiveness of real-world commodity trade and storage. Thus, we present a model of oligopolistic commodity speculation, in which a limited number of speculators practice non-cooperative storage in an infinite horizon game. A significant technical difficulty due to the non-negative stock constraint is overcome, and a tractable sub-game perfect equilibrium is presented, in which it is shown that less is stored and prices are more volatile than under perfect competition. It turns out that a tax on consumption of the good would increase storage, stabilize prices, increase welfare and raise speculative profits; the oligopolists would thus lobby for a tariff raised against their own shipments.
\end{abstract}

JEL Classification numbers: (022), (026), (611), (715)

Keywords: Commodity prices, Dynamic oligopoly, Speculation, Strategic tariffs 


\section{Introduction.}

This paper offers a contribution to the theory of speculative equilibrium in markets for storable commodities.

Great strides have been made over the past decade in the theory of stochastic and dynamic equilibrium in primary commodity markets. One large impetus in this direction was the work of Newbery and Stiglitz (1981); developments have been made by, for example, Scheinkman and Schechtman (1983), Newbery (1984), Deaton and Laroque (1991) and in various work by Williams and Wright, largely surveyed in Williams and Wright (1991). However, the relationship between storage and market structure has not been much explored. The overwhelming majority of existing studies assume perfect competition. Two exceptions are Newbery (1984) and Williams and Wright 1991 , ch.11). Newbery explores dominant producer equilibria, in which one large producer/speculator exploits its market power while a submissive competitive fringe accepts zero storage profits and takes price as given. Williams and Wright survey the theory of monopoly in storage. There appears to be little work examining the consequences of restricted entry into the business of commodity trade or storage or of strategic competition among primary commodity speculators. The nearest approximations are in the theory of oligopolistic extraction of a natural resource (e.g., Reinganum and Stokey (1985), Sundaram (1989) and especially Loury (1990) which can be read as a model of strategic storage). However, this literature does not have to cope with the issues of random harvests and stockouts which are central to many agricultural commodities. 
This paper represents a step into that field, with a stochastic model of an n-member speculation oligopoly. It is a quite simplified model. There are no futures. Storage and speculation are treated as the same thing, and the speculators are the only middlemen between producers and consumers. Nonetheless, the model is not easy to solve, and since in real markets it is difficult to separate storers, speculators and traders, it is hoped that it may shed some useful insight on some actual markets.

The idea of storage or speculation as being carried out by an oligopoly has a quite plausible ring in some primary commodity markets. Trade in some of these markets is highly concentrated, and there can be important fixed costs to entry. Some accounts of the world grain trade, for example, stress the importance of large fixed costs in storage and transport facilities which must be incurred in order to compete and which contribute to the dominance of that trade by five very large firms. For example, Morgan $(1979$, p.97) describes the massive elevator at Baie-Comeau, Quebec and ancillary transport infrastructure which Cargill had to construct in 1959 before it was able to compete in the European market. The same author argues that Cargill and a competitor, Continental, controlling one half of the grain storage space in U.S. ports between them, have genuine market power in grain storage (p. 235). Similar observations have been made about a variety of tropical commodities in which trade has long been highly concentrated (for example, Bauer (1953) and Maizels (1984, pp. 28-9)).

Further, evidence on the returns to speculation in primary goods seem to show that the large players have tremendous informational advantages over small ones. This was shown with futures data by Houthakker (1957), who concluded that only large traders could forecast commodity prices at all. Hartzmark (1987), with daily data on the individual positions of more than 5,000 
traders on a variety of commodities, found that only the large ones even broke even and only those who dealt in the good commercially as well as trading it in futures made any systematic positive returns. In addition, $58 \%$ of the profits were made by only five of the traders. Thus, it seems that only a handful of traders have the information network to speculate successfully. The creation of this network entails a second kind of fixed cost of entry ${ }^{1}$.

As a theoretical matter, if oligopoly is ruled out erroneously, the nature of the equilibrium predicted may be qualitatively as well as quantitatively wrong. Both monopoly and perfect competition (see Scheinkman and Schechtman (1983) and Deaton and Laroque (1991)) typically yield unique equilibria through contraction mapping arguments, but one of the great themes of recent oligopoly research is the multiplicity of dynamic equilibria they generally offer including collusion and its collapse, price wars and so forth (see Shapiro, 1988, for a survey). Thus, there could be patterns in time series for commodity prices that simply do not make sense without imperfect competition. However, this is merely a conjecture and is beyond the scope of this paper.

A serendipitous benefit of this research is a method of solving linear-quadratic games with a constraint which might prove useful in the theory of production oligopoly with storable output. Here, the constraint is the non-negativity of stocks. Usually, infinite horizon oligopoly models

\footnotetext{
${ }^{1}$ The need to build an information network to compete effectively fits into the "endogenous sunk cost" paradigm of Sutton (1991), contrasted with exogenous sunk costs such as fixed costs incurred in building an elevator. Sutton (Ch. 3) shows that if the important sunk costs are endogenous, a market can remain highly concentrated even as demand grows arbitrarily large and technology is held fixed, because a rise in demand, rather than encouraging entry, raises equilibrium investments by the incumbents, thus also raising the cost of entry for a potential entrant. This may be the most plausible explanation for the high levels of concentration in trade in certain commodities, where investments in network building probably greatly exceed fixed costs in storage.
} 
need to allow for negative inventories to get any results to mention (e.g., Judd (1990), Kirman and Sobel (1971)) because with a constraint that sometimes binds, even a linear-quadratic model becomes unmanageable by analytic methods. Here we apply a trick from competitive commodity theory that makes things work out neatly. Whether or not it has other uses remains to be seen.

Section 2 reviews perfectly competitive storage equilibria with linear demand; Section 3 takes the same setting with a fixed set of speculators and studies a game theoretic equilibrium; Section 4 studies some special properties of the equilibrium, including the striking "independence" property whereby rational speculators "ignore" each other. Section 5 discusses the assumption of fixed storage capacities, and the difficulties in relaxing it. Section 6 derives some implications of the model for the time path of prices, and gives numerical examples. Section 7 concludes. 


\section{A Review of Perfect Competition.}

We use essentially the same setting as Newbery (1984), and for ease of comparison will use much of the same notation. We assume a market with $\mathrm{n}$ speculators, who face a constant linear demand schedule so that the price of the good in period $t$ is $p_{t}=a-b C_{t}$, where $a, b>0$ and $C_{t}$ is the amount sold to consumers in period t. The crop is harvested once a year and follows a simple i.i.d. process: a "normal" crop $\mathrm{h}^{+}$is reaped with probability $\rho$ and a "drought", with crop $h^{-}<h^{+}$occurs with probability $1-\rho$. We will denote the average crop as $\bar{h}$. The producers are perfectly competitive and separate from the speculators; it is best to think of a large number of small farmers and consumers who together with the $\mathrm{n}$ speculators buy and sell the good on an anonymous auction market.

The speculators have a storage technology as follows: speculator $\mathrm{j}$ can store a quantity of inventories no less than zero and no greater than her capacity constraint $\mathrm{K}^{j}$. There is a financial cost of $\mathrm{k}$ per unit held from one period to the next, and in the interval a fraction $\delta$ will spoil. Speculators do not have the option of burning or discarding stocks (perhaps $\delta$ includes the cost of disposing of goods that have "turned", but other stocks may not be discarded). Finally, all speculators are risk neutral, and have a discount rate equal to $\beta$.

The first question of interest is: what would happen if, instead of having the number of speculators fixed at $\mathbf{n}$, there was free entry into the sector and all speculators took price as given? Clearly profits from speculation would be driven to zero, and hence the definition of equilibrium: DEFINITION: A function $\varphi: D \times\left\{h^{-}, h^{+}\right\} \rightarrow \mathbb{R}_{+}$, with $\mathrm{D} \subset[0, \mathrm{~K}]$ and $\mathrm{I} \in \mathrm{D}$ implying $(\mathrm{I}-\delta) \varphi(\mathrm{I}, \mathrm{h}) \in \mathrm{D}$, is an equilibrium iff: 


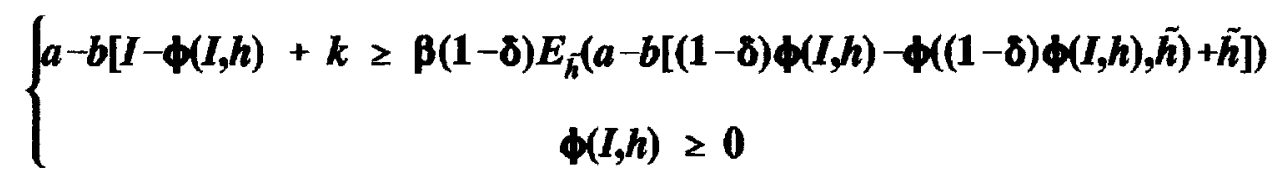

for all $\mathrm{I} \in[0, \mathrm{~K}]$ and for $\mathrm{h}=\mathrm{h}^{+}$or $\mathrm{h}^{-}$, where $\mathrm{E}_{\tilde{\mathrm{h}}}$ stands for expectations with respect to the harvest, $\mathrm{K}$ is the aggregate capacity constraint, and whenever one of these inequalities holds strictly, the other must hold with equality.

The function $\phi$ gives the equilibrium level of storage. Inequalities (2.1) guarantee that the cost of investing in another unit of stocks, $p_{t}+k$, is always at least as great as the expected revenue it would bring, $\beta(1-\delta) \mathrm{p}_{\mathrm{t}+1}$, so that profits can never be positive in expectation. Further, if speculators strictly prefer to dump stocks (i.e., the first inequality holds strictly), they will not carry any out; and if they carry stocks out (i.e., the second inequality holds strictly), then they must be indifferent between doing so and not doing so (the first becomes an equality). The following result is a slightly generalized version of results in Newbery (1984).

Proposition 1. Let

$$
\begin{aligned}
\alpha & =\frac{1+\beta(1-\delta)^{2}-\left(\left(1+\beta(1-\delta)^{2}\right)^{2}-4 \beta(1-\delta)^{2} \rho\right\}^{1 / 2}}{2 \beta(1-\delta)^{2} \rho} \\
z_{t} & =I_{t}+h_{t} \forall t, \\
\bar{z} & =(1-\beta(1-\delta) \rho \alpha)^{-1}\left[(1-\beta(1-\delta)) a / b+\beta(1-\delta) \bar{h}-\alpha \beta(1-\delta) \rho h^{+}+k / b\right] \\
z_{\max } & =(1-(1-\delta) \alpha)^{-1}\left(h^{+}-(1-\delta) \alpha \bar{z}\right), \text { and } \\
D & =\left[0, \alpha\left(z_{\max }-\bar{z}\right)\right] .
\end{aligned}
$$


Then if

$$
(1-\delta) \alpha\left(z_{\max }-\bar{z}\right)+h^{-} \leq \bar{z} \leq h^{+}
$$

and $\alpha\left(\mathrm{z}_{\max }-\overline{\mathrm{z}}\right) \leq \mathrm{K}$, the function $\varphi(\mathrm{I}, \mathrm{h})=\max [0, \alpha(\mathrm{z}-\overline{\mathrm{z}})]$ is an equilibrium.

Proof: It is easy to verify that $\alpha<1$, so the difference equation implied by $\varphi$ as the stocking function in year of a normal harvest, namely :

$\mathrm{z}_{\mathrm{t}+1}=\mathrm{I}_{\mathrm{t}+1}+\mathrm{h}^{+}=(1-\delta) \alpha\left(\mathrm{z}_{\mathrm{t}}-\overline{\mathrm{z}}\right)+\mathrm{h}^{+}$, is stable with steady state $\mathrm{z}_{\max }$. If the system starts with $\mathrm{z}_{\mathrm{t}}$

$\leq z_{\max }$, then, $z_{\max }$ will indeed be the supremum for $z$. (So $I_{t} \in D$ implies $I_{t+1} \in D$, as required.) Thus the highest possible value of $\mathrm{z}$ during a drought is $(1-\delta) \alpha\left(\mathrm{z}_{\max }-\overline{\mathrm{z}}\right)+\mathrm{h}^{-}$, so that (2.2) implies that regardless of $\mathrm{z}$, during a drought there will be no carryout. Further, $\mathrm{h}^{+}>\overline{\mathrm{z}}$ implies that even with no carryin, there will always be carryout if there is a normal harvest. Thus $E_{\tilde{h}}[f(z, \tilde{h})]=$ $\rho \alpha(z-\bar{z})$ regardless of $z$. Plugging this into the definition of equilibrium gives, in cases when $h$ $=\mathrm{h}^{+}$and thus $\mathrm{I} \neq 0$,

$$
\begin{aligned}
& \alpha(z-\bar{z})= \\
& \frac{\left(b z-(1-\beta(1-\delta)) a-\beta(1-\delta) b\left[\bar{h}-\rho \alpha\left(h^{+}+(1-\delta) \alpha z-(1+(1-\delta) \alpha) \bar{z}\right)\right]-k\right.}{b\left(1+\beta(1-\delta)^{2}\right)}
\end{aligned}
$$

This is a straight line in $z$ on both sides of the equation. The intercepts can easily be checked to be equal by setting $\mathrm{z}=0$, and matching the slope coefficients gives: 


$$
\begin{gathered}
\alpha=\frac{b+\beta(1-8) b \rho \alpha(1-8) \alpha}{b\left(1+\beta(1-\delta)^{2}\right)} \\
\text { or } \beta(1-\delta)^{2} \rho \alpha^{2}-\left(1+\beta(1-\delta)^{2}\right) \alpha+1=0 .
\end{gathered}
$$

$\alpha$ as defined is clearly a solution to this equation. (Note that the other root could not do the same job because, as is easily checked, it is greater than one and would cause explosive behaviour.) The other properties are straightforward. Q.E.D.

Note that there is no guarantee that the equilibrium will have this form if the system is started far above $z_{\max }$ (i.e., if $z_{1} \notin D$ ). The secret of the simplicity of the stocking function is in the fact that in the indicated range it is an exogenous variable, the harvest, that determines whether the system will stock out or not. Speculators accumulate stocks in good years and dump them all during droughts.

A final note on the competitive equilibrium: it is unique. This is established in Scheinkman and Schechtman (1983) and Deaton and Laroque (1991) for a much broader class of demand functions than we consider here.

\section{The Speculation Game.}

Now we fix the number of speculators at $\mathrm{n}$, and divide up the industry storage capacity $\mathrm{K}$ among them. We presume that they are aware of each other's holdings so that the inventory vector $I_{t}=\left[I_{t}^{1}, I_{t}^{2}, \ldots, I_{t}^{n}\right]^{\prime}$ is always common knowledge. Equilibrium is now defined as follows: 
DEFINITION. A vector of value functions $\mathrm{V}^{\mathrm{j}}: \mathrm{D} \times\left\{\mathrm{h}^{-}, \mathrm{h}^{+}\right\} \rightarrow \mathbb{R}, \mathrm{j}=1, . ., \mathrm{n}, \mathrm{D} \subset\left\{\mathrm{I}: \mathrm{I}^{\mathrm{j}} \in\left[0, \mathrm{~K}^{\mathrm{j}}\right] \forall \mathrm{j}\right\}$, will be called an equilibrium iff for any $I \in D$ and for either $h$ there exist sales decisions $s^{1}, . ., s^{n}$ (with $\left.s=\left[s^{1}, . . s^{n}\right]^{\prime}\right)$ such that :

(3.1)(a) $\quad \mathrm{s}^{\mathrm{j}} \leq \mathrm{I}^{\mathrm{j}} \forall \mathrm{j}$;

(3.1)(b) $\quad \sum_{\mathbf{j}=1}^{n} s^{j}+h \geq 0$;

(3.1)(c) $\quad \mathrm{s}^{\mathrm{j}} \geq \mathrm{I}^{\mathrm{j}}-\mathrm{K}^{\mathrm{j}} \forall \mathrm{j}$

$(3.1)(d) \quad$ For each $J, s^{J}\left(a-b\left[\sum_{j=1}^{n} s^{j}+h\right]\right)-k\left(I^{J}-s^{J}\right)+\beta E_{\hat{h}}\left[V^{J}((1-\delta)(I-s), \tilde{h})\right] \geq$ $\bar{s}\left(a-b\left[\sum_{j \neq J} s^{j}+\bar{s}+h\right]\right)-k\left(I^{J}-\bar{s}\right)+\beta E_{\hat{h}}\left[V^{J}\left((1-\delta)\left(I^{1}-s^{l}\right), . .,(1-\delta)\left(I^{J}-\bar{s}\right), . .(1-\delta)\left(I^{n}-s^{n}\right), \tilde{h}\right)\right]$

for any $\bar{s} \in\left[-\left(\sum_{j}, z_{J} s^{j}+h\right), I^{J}\right] \cap\left[I^{J}-K^{J}, I^{J}\right]$.

(3.1)(e) For each j, $V^{j}(I, h)=s^{j}\left(a-b\left[\sum_{j=1}^{n} s^{j}+h\right]\right)-k\left(I^{j}-s^{j}\right)+\beta E_{h}\left[V^{j}((1-\delta)(I-s), \tilde{h}]\right.$.

(3.1)(f) $\quad I \in D$ implies $(1-\delta)(I-s) \in D$.

Evidently the problem is a bit more complicated than the perfectly competitive one, but these six conditions are all commonsensical. (3.1)(a) states that a speculator may not sell more than she has on hand (or, put differently, may not choose to hold negative inventories). (3.1)(b) says that speculators can not buy anything more than the whole crop. $(3.1)(\mathrm{c})$ bounds purchases with the capacity constraint. (3.1)(d) is the first significant economic condition: it says that the expected present value of profits of each speculator must be maximized given the understood behaviour of the others; that at each moment speculation takes the form of a Nash equilibrium in sales. Note the anatomy of this condition: $s^{J}\left(a-b\left[\sum_{j=1}^{n} s^{j}+h\right]\right)$ is current revenue from sales (possibly negative); $\mathrm{k}\left(\mathrm{I}^{\mathrm{J}}-\mathrm{s}^{\mathrm{J}}\right)$ is the cost of holding the new level of stocks over to the next period; and $\beta \mathrm{E}_{\tilde{h}}\left[\mathrm{~V}^{\mathrm{I}}((1-\delta)(\mathrm{I}-\mathrm{s}), \tilde{\mathrm{h}})\right]$ is the discounted expected present value of the operation next period, taking into account the revised level of stocks. The constraint on $\bar{s}$ in $(3.1)(d)$ is just the 
implication of (3.1)(a-c). Finally, (3.1)(e) guarantees that these value functions really do give the expected present value of the income stream from speculation. It is the key recursive condition that makes solution of this game possible. (Condition (3.1)(f) simply ensures that the value functions are defined on any part of the state space that the equilibrium leads to.)

Thus, one can think of equilibrium in either of two ways: as the simultaneous solution of $\mathrm{n}$ Bellman equations, each taking the solution of the other $n-1$ as given; or as a Nash equilibrium of a two-period model, which thus maps the initial vector of value functions into a new one, with the final equilibrium being a fixed point of this mapping. Importantly, the fact that this definition insists on a Nash equilibrium at each value of $I \in D$ and both values of $h$ means that the equilibrium is not merely Nash, but subgame perfect.

Now note that the process by which $I_{t}$ is transformed into $I_{t+1}$ is linear and the return to each speculator (see $(3.1)(\mathrm{d})$ ) is quadratic in $\mathrm{I}_{\mathrm{t}}$. This suggests that we might be able to use the well-known apparatus of linear-quadratic games ${ }^{2}$ in order to derive a solution elegantly, with linear functions governing equilibrium motion of sales and stocks. The problem with this is the non-negativity constraint on stocks. This means that when inventories are near zero, a bad harvest will be likely to drive them right down to zero; thus in that region equilibrium carryout will be independent of carryin, while elsewhere they will be a non-trivial linear function of the carryin. Thus, the equilibrium behaviour can be at best piecewise linear, and the hope of recovering anything simple in recursions as in (3.1)(e) is dim. It is because of the violence non-negativity does to tractable linear-quadratic games that Judd (1990) decided not to rule out

${ }^{2}$ For a good discussion of general linear-quadratic dynamic programs, see Sargent (1987, ch. 1). They are easily adapted to games; a general treatment is Kydland (1975). See Judd (1990) for an application. 
negative inventories in his dynamic oligopoly model, preferring to find equilibria in which there would be no stockouts, but that is not an option here, because in the study of commodity prices the occurrence of stockouts is a large part of the interest. Further, it is widely known in game theory that what could happen but does not -- events off of the equilibrium path -- could affect what happens on the equilibrium path.

The solution we will explore here is inspired by the Newbery equilibrium discussed above: we will hope to find a region of $\mathbf{R}^{\mathrm{n}}$, into which the vector of inventories will permanently settle, in which the system will move between the two regimes -- stockout and carryout -- on the force of the weather alone. That way we might pray that the value function will be "almost quadratic", as:

$$
V^{j}(I, h)=\left\{\begin{array}{l}
x^{\prime} E^{j} x \text { if } h=h^{+} \\
x^{\prime} F^{j} x \text { if } h=h^{-}
\end{array}\right.
$$

where $x=\left[1, I^{1}, I^{2}, \ldots, I^{n}\right]^{\prime}$ and $E^{j}$ and $F^{j}$ are $(n+1)$-square matrices. (Note the constant in the state vector.) It turns out that indeed in a wide variety of cases such equilibria exist. The following proposition establishes a (cumbersome) sufficient condition which will be used later.

Proposition 2. Let $e_{2}$ be negative and satisfy:

$$
\begin{aligned}
\beta^{2}(1-\delta)^{4} \mathrm{e}_{2}^{3}- & {\left[2 \mathrm{~b} \beta(1-\delta)^{2}-b \beta^{2}(1-\delta)^{4}\right] \mathrm{e}_{2}^{2}+\left[\mathrm{b}^{2}-\beta \rho(1-\delta)^{2} \mathrm{~b}^{2}\right.} \\
- & \left.2(1-\rho) \mathrm{b}^{2} \beta(1-\delta)^{2}\right] \mathrm{e}_{2}+(1-\rho) \mathrm{b}^{3}=0 .
\end{aligned}
$$

Also let $\mathrm{e}_{2}^{+}, \mathrm{e}_{2}^{-}, \mathrm{g}, \mathrm{e}_{1}, \mathrm{e}_{1}^{+}, \mathrm{e}_{1}^{-}, \mathrm{f}, \mathrm{f}^{-}, \mathrm{e}_{0}, \mathrm{e}_{0}^{+}$, and $\mathrm{e}_{0}^{-}$be as given in Table I. Then if: 
(3.2)(h)

$$
\frac{a-b h^{-}+k-\beta(1-\delta) e_{1}}{b(n+1)} \geq K^{j} \geq-\frac{2(1-\delta) f}{(1-(1+2 g)(1-\delta))(n+1)} \forall j
$$

and if $f<0$, the value functions:

$$
V^{J}(I, h)=\left\{\begin{array}{l}
e_{0}^{+}+e_{1}^{+} I^{J}+e_{2}^{+} I^{J} \sum_{j=1}^{n} I^{j} \text { if } h=h^{+} \\
e_{0}^{-}+e_{1}^{-} I^{J}+e_{2}^{-} I^{J} \sum_{j=1}^{n} I^{j} \text { if } h=h^{-}
\end{array}\right.
$$

constitute an equilibrium, with $s=2 f /(n+1) 1-2 g I$ if $h=h^{+}$and $s=I$ if $h=h$, where 1 is a vector of ones.

Note: It is easy to see that $e_{1}=\rho e_{i}^{+}+(1-\rho) e_{i}^{-}$for $i=0,1,2$. Thus, $E_{h}\left[V^{J}(I, h)\right]=e_{0}+e_{1} I^{J}+$ $e_{2} I^{J} \sum_{j-1}^{n} I^{j}$. Also note that $V^{J}\left(I, h^{-}\right)=I^{J}\left(a-b\left[\sum_{j=1}^{n} I^{j^{j}}+h^{-}\right]\right)+\beta e_{0}$, which is the revenue to $J$ given a general stockout plus discounted expected future earnings. Note also that we will assume throughout that there are at least two speculators; similar reasoning works for a monopolist but that case will be deferred.

Proof: See Appendix.

What this propostion says is that under certain conditions a quite simple system of behavioural rules will constitute a subgame-perfect equilibrium of this game. In a good year, each speculator sells an amount given by a straight-line function of her own stocks and nothing else, and in a drought all speculators sell everything. The intercept of the sales function is negative, so that when stocks are low and the harvest is normal, speculators buy; and its slope 
is between zero and one, so that after a large number of consecutive good harvests stocks converge to a maximum. Thus, the equilibrium looks much like the competitive one.

At this stage, it is not clear that any useful information is contained in Proposition 2. Most of the conditions it assumes are unintuitive and, especially the cubic equation on which they all hinge, intractable. How are we to know if there are any roots that, filtered through the complex of definitions in Table I, will satisfy the key inequalities? Is there an easy way of finding these roots? And what will be the relationship of this equilibrium to the competitive one? The answers are found in the following three propositions, which show how simple the problem really is.

Proposition 3. Let the conditions of Propositions 1 and 2 hold. Let $\alpha$ be as given in Proposition 1 and $\mathrm{g}$ be as in Proposition 2. Then $\alpha=1+2 \mathrm{~g}$.

Proof. Rearranging (3.2)(b) gives:

$$
e_{2}=\frac{b}{\beta(1-\delta)^{2}(1+2 g)}=-\frac{b \quad(1-d)}{\beta(1-\delta)^{2} d}
$$

where $d=1+2 g$.

Combining (A.1) from the proof of Proposition 2 with (3.2)(a) gives:

$$
e_{2}=\rho \beta e_{2}(1-\delta)^{2}(1+2 g)^{2}-4 \rho g^{2} b-(1-\rho) b, \text { so }
$$




$$
e_{2}=-\frac{\left((d-1)^{2} \rho+1-\rho\right) b}{\left(1-\beta \rho(1-\delta)^{2} d^{2}\right)}
$$

Putting these two together yields:

$$
\frac{(1-d)}{\beta(1-\delta)^{2} d}=\frac{\left((d-1)^{2} p+1-\rho\right)}{\left(1-\beta \rho(1-\delta)^{2} d^{2}\right)}
$$

Thus,

$$
\begin{gathered}
(1-d)\left(1-\beta \rho(1-\delta)^{2} d^{2}\right)=\beta(1-\delta)^{2} d(d-1)^{2} \rho+(1-\rho) \beta(1-\delta)^{2} d, \text { so } \\
1-\beta \rho(1-\delta)^{2} d^{2}-d+\beta \rho(1-\delta)^{2} d^{3} \\
=\beta(1-\delta)^{2} \rho d^{3}-2 \beta(1-\delta)^{2} \rho d^{2}+\beta(1-\delta)^{2} \rho d+(1-\rho) \beta(1-\delta)^{2} d .
\end{gathered}
$$

The cubic terms cancel. The equation then becomes:

$$
\beta \rho(1-\delta)^{2} d^{2}-\left(1+\beta(1-\delta)^{2}\right) d+1=0 .
$$

But this is exactly equation (2.3), which determined $\alpha$. Since we know (by (3.2)(b) and the negativity of $e_{2}$ ) that $d=1+2 \mathrm{~g}$ is less than one, $1+2 \mathrm{~g}$ is equal to the smaller root of this equation. But that is $\alpha$. Q.E.D.

What this proposition tells us is that it is simple to calculate $g$, and from that, $e_{2}$ (by (3.2)(b)), and from that, everything else. More importantly for the economics of the matter, we have, quite strikingly, the same marginal propensity to sell out of stocks for perfectly competitive speculation as for the speculation oligopoly, and that regardless of the number of speculators, as long as there are at least two. Similarly, since $(1-\delta)(1+2 g)=(1-\delta) \alpha$ is the autoregression parameter for stocks in periods of normal harvest, these stocks will have exactly the same persistence properties whether there are two speculators or a hundred thousand. The same is true, 
clearly, for $e_{2}$ and (by (3.2)(c)) for $e_{1}$.

Now, note that in both equilibria discussed so far aggregate sales by speculators in a normal year take the form of a constant straight-line function of stocks carried in. The intercept is of course negative in both cases. This proposition relates the two.

Proposition 4. The intercept of the normal-year aggregate sales function for an equilibrium of the type described in Proposition 2 is always equal to $n /(n+1)$ of the competitive intercept

Proof: In the competitive case, carryout at the end of a normal year when there was zero carryin is $\alpha\left(h^{+}-\bar{z}\right)$. Therefore, the competitive sales intercept is

$$
-\alpha\left(h^{+}-\bar{z}\right)=\alpha \frac{1}{(1-\beta \rho \alpha(1-\delta))}\left[(1-\beta(1-\delta)) \frac{a}{b}-h^{+}+\frac{k}{b}+\beta(1-\delta) \bar{h}\right] .
$$

To find the intercept for the case of oligopoly, recall that each speculator's sales function in Proposition 2 had an intercept of $2 f /(n+1)$, so the aggregate intercept is $2 n f /(n+1)$. Recalling the definition of $f$, this equals:

$$
\begin{gathered}
\frac{n \quad\left(a-b h^{+}+k-\beta(1-\delta) e_{1}\right)}{(n+1) \quad\left(b-\beta(1-\delta)^{2} e_{2}\right)} \\
=\frac{n \quad\left(a-b h^{+}+k-\beta(1-\delta) e_{1}\right)}{(n+1)\left[b-\beta(1-\delta)^{2} \frac{b(\alpha-1)}{\beta(1-\delta)^{2} \alpha}\right]}
\end{gathered}
$$


(by (3.2)(b) and Proposition 3)

$$
\begin{array}{r}
=\frac{\alpha n}{(n+1)}\left[\frac{a}{b}-h^{+}+\frac{k}{b}-\frac{\beta(1-\delta) e_{1}}{b}\right] \\
=\frac{\alpha n}{(n+1)}\left[\frac{a}{b}-h^{+}+\frac{k}{b}-\frac{\beta(1-\delta)}{(1-\beta \rho \alpha(1-\delta))}\left[\frac{a}{b}-\bar{h}-\alpha \rho\left(\frac{a}{b}-h^{+}+\frac{k}{b}\right)\right]\right] \text { by (3.2)(c) } \\
=\frac{\alpha n}{(n+1)(1-\beta \rho \alpha(1-\delta))}\left[(1-\beta(1-\delta)) \frac{a}{b}-h^{+}+\frac{k}{b}+\beta(1-\delta) \bar{h}\right]
\end{array}
$$

$=\mathbf{n} /(\mathbf{n}+1)$ times the competitive intercept. Q.E.D.

Thus the intercept is farther from zero (smaller) the more speculators there are, with the limit as $n \rightarrow \infty$ being the competitive case. Thus the competitive speculators will always buy more, or sell less, than the imperfectly competitive, given the same level of inherited stocks. In addition, for any set number of consecutive good harvests the competitive speculators will have $(\mathrm{n}+1) / \mathrm{n}$ times the stocks that the oligopolists would.

Proposition 5. The expected discounted profits of each oligopolistic speculator in the period after a drought are strictly positive. They are strictly decreasing in the number of speculators, and take a limit of zero as $\mathrm{n} \rightarrow \infty$. Furthermore, the aggregate speculative profits also decline to zero as the market becomes crowded. Precisely, 


$$
e_{0}=\frac{2 \rho \alpha\left(a-b h^{+}-\beta e_{1}(1-\delta)\right)^{2}}{(1-\beta) b(n+1)^{2}},
$$

so that $\mathrm{e}_{0}$ and $\mathrm{ne}_{0} \rightarrow 0$ as $\mathrm{n} \rightarrow \infty$.

Proof. From (3.2)(f),

$$
\begin{gathered}
e_{0}=\frac{2 f \quad \rho}{(n+1)(1-\beta)}\left\{a-b\left(\frac{2 n f}{(n+1)}+h^{+}\right)+k-\beta e_{1}(1-\delta)+\beta e_{2}(1-\delta)^{2} \frac{2 n f}{(n+1)}\right\} \\
=\frac{2 f \quad \rho}{(n+1)(1-\beta)}\left\{a-b h^{+}+k-\beta e_{1}(1-\delta)-\left(b-\beta e_{2}(1-\delta)^{2}\right) \frac{2 n f}{(n+1)}\right\} \\
=\frac{\rho \quad 2 \quad\left(a-b h^{+}+k-\beta e_{1}(1-\delta)\right)^{2}}{(1-\beta)(n+1)^{2}}\left(b-\beta e_{2}(1-\delta)^{2}\right)
\end{gathered}
$$

Q.E.D.

Finally, we complete the spectrum of market concentration:

Proposition 6. All of the results on oligopoly hold true for a monopoly if we plug $n=1$ into the above formulae. 
Proof: Consider the value function:

$$
V(I, h)=\left\{\begin{array}{c}
e_{0}^{+}+e_{1}^{+} I+e_{2}^{+} I^{2} \text { if } h=h^{+} \\
I^{J}\left(a-b\left[I+h^{-}\right]\right)+\beta \rho e_{0}^{+} /(1-(1-\rho) \beta) \text { if } h=h^{-}
\end{array}\right.
$$

where I is now a scalar, the monopolist's holdings. Solving the first-order conditions for Bellman's equation leads straightforwardly to $s=f-2 g I$, with $f$ and $g$ defined as above. This is the non-cooperative sales function derived above, with $n=1$ plugged in. Putting this into Bellman's equation then gives us a quadratic function whose coefficients can be matched with those of the value function, just as in the proof of Proposition 2. We recover the matching equations from that proof, (A.1), (A.2) and a version of (A.3), with $n=1$ immediately. Finally, plugging $n=1$ into $(3.2)(\mathrm{h})$ turns it into:

$$
\frac{a-b h^{-}+k-\beta(1-\delta) e_{1}}{2 b} \geq K \geq-\frac{(1-\delta) f}{(1-\alpha(1-\delta))}
$$

Since the expression on the right hand side is the maximum stock of inventories the monopolist would ever accumulate, the monopolist satisfying (3.2)(h) would never violate its capacity constraint. Further, in a drought the monopolist's first order condition is satisfied by $\mathbf{s}$ $=\mathrm{f}^{-}-2 \mathrm{gI}$, which implies a stockout iff $\mathrm{s} \geq \mathrm{I}$, i.e., iff $\mathrm{I} \leq \mathrm{f}^{-} /(1+2 \mathrm{~g})$, which is the expression on the left hand side. Thus, the inequality ensures that the monopolist will indeed stock out if and only if there is a bad harvest, confirming that the proposed value function is the right one. Q.E.D. 
To sum up, despite the potential complexity in looking for a subgame perfect equilibrium with an infinite horizon, in which every possible contingency of every possible future must be embodied in each move, and despite the real complexity of some technical preliminaries in Proposition 2 , the equilibrium finally takes a form of terrible simplicity. The speculators sell all in a drought and accumulate in good years, following a simple linear rule that leads them to buy less each succeeding good year until they converge to a maximum. The slope of this linear rule is the same regardless of the number of speculators, even for a monopoly, and it, as well as all other coefficients of equilibrium, has a quite simple closed form expression.

\section{The "Independence" Property and other features.}

Perhaps the most striking feature of the equilibrium developed above is that in it each speculator persistently ignores the others. The sales function for a good year is, we recall, $\mathrm{s}=$ $2 f /(n+1) 1+(1-\alpha) I$. There is no interaction between neighbor's stocks and own purchases; only own stocks have any effect. This is the reverse of what one might expect, on the intuition that a neighbor weighed down with stocks would be keen to dump some of them now, thus making now a poor time to earn revenue from one's own stocks, and thus depressing own current sales. Why is this not true?

Recalling from the proof of Proposition 2 the solution to 1 's first order condition, namely

$$
s^{1}=f-g\left(I^{1}+\sum_{j=1}^{n} I^{j}\right)-(1 / 2) \sum_{j \neq 1} s^{j}
$$

we can see that the reasoning is half right. Ceteris paribus, an increase in the (expected) sales of others will lead 1 to reduce her own sales that period, by half of the increase. But holding 
sales constant, the stocks of others have their own positive effect on sales (recall $\mathrm{g}<0$ ). Noting that $e_{2}$ is the expected derivative of the marginal value of own stocks with respect to other stocks, and reviewing the definition of $g((3.2)(b))$, we can see that $-\mathrm{g}$ is a measure of the weakening of 1 's incentive to carry stocks into the next period due to an increase in the expected carryout of others. This future effect comes from exactly the same source as the current sales effect; in fact, it is the same effect, telescoped into the future along with the repercussions of the increased inventories extending possibly infinitely far into the future. Thus, we have from the increase in $\sum_{\mathrm{j} \neq 1} \mathrm{I}^{\mathrm{j}}$ two conflicting forces, one arguing for clinging to stocks and the other for dumping them now. These annihilate each other. 1 is worse off but has nothing to do about it.

A second feature of the equilibrium that bears special mention is its very marked tendency toward equality. When the weather is good all speculators accumulate and in time approach their respective limits -- but since they all have the same limit, their differences wither away. One might think that if one speculator had begun for whatever reason with eighty percent of total stocks then he would continue to dominate the market, but in this equilibrium with good weather that dominance would slowly be eroded, and with one bad year it would disappear overnight. This is illustrated for a speculative duopoly by figure 1. Figure 1(a) shows stock dynamics during normal years and $1(\mathrm{~b})$ during droughts. In either case the arrows are always moving toward the $45^{\circ}$ line. (Note that Figure 1 also illustrates (3.2)(h). The region under the heavy line is where

$\sum_{j=1}^{n} I^{j}+I^{j} \leq 2 f^{-} /(1+2 g) \equiv \kappa, J=1,2$, which we may recall from the proof of Proposition 2 is the region in which stockouts are an equilibrium during droughts.)

This inexorable march toward equality provides a strong contrast with the competitive 
model, which, of course, makes no prediction at all about the distribution of stocks. It is also one feature of the present model that does not seem to resemble the real world very much.

No attempt is made here to try to relate either of these properties to a lack of convexity in demand. It is an obvious place to look for robustness. For example, there appear to be large differences between the slope of the competitive $\varphi$ function and that of the carryout function for a monopolist in the case of isoelastic demand (see Williams and Wright, 1991, ch. 11), in contrast to our finding of identical slopes.

\section{The Case without Capacity Constraints.}

The reader may well wonder why we bothered mentioning capacity constraints, since they do not seem to affect anything. For example, in Figure 1, the capacity constraint vector can be anywhere in the broken-line box between $I_{\max }$ and $\kappa / 3$ without changing anything about the equilibrium at all. The answer has to do with the requirements of subgame perfection, and of economic rationality.

If there were no capacity constraints, then generally (as we will see in numerical examples) it will be possible for one speculator to buy enough stocks to so that next period carryin will lie outside of the heavy line in Figure 1 , in other words, in a region in which $\sum_{j=1}^{n} I^{j}+I^{J}$ exceeds $\kappa$. The question is, if it is possible, but if everyone else plans to stay inside the region of interest, is it desirable? If the answer is "No", then our whole argument and set of propositions are intact. It seems implausible that the answer could be otherwise, since even within the region under the heavy line, stocks above $I_{\max }$ drift back toward $I_{\max }$; there is no tendency of this system to reach 
upward and bang against the capacity constraint. Would removing the constraint, then, change that?

The answer is uncertain. The problem is that when one allows a speculator to leave that region, one must ask what would happen if she actually did, in order to ask whether or not it would be optimal to do so. Thus one needs to extend the value function to all points that are accessible to speculators from under the heavy line. It appears to be a difficult thing to do.

Call the region under the heavy line $R(1)$. Then one approach is to assume that one knows that there is a region beyond the heavy line, say $R(2)$, such that if $I \in R(2)$ then in a drought I will move into $R(1)$ and in a normal year I will stay in $R(2)$. Notice that the guess is that there is no stock-out in a drought year, since the original value function was defined by the assumption of stockouts in bad years, and $\mathrm{R}(1)$ encloses all of those points for which stockouts are an equilibrium in bad years. By this method, one can calculate a value function for $R(2)$ and try to figure out whether or not it will be optimal for any speculator to move there given that no one else is doing so.

But then one must verify that, once in $R(2)$, it really will be optimal to descend to $R(1)$ if everyone else is instead of heading up to a higher level still. This verification means extending the value function to some $\mathbf{R}(3)$, etc.

Thus, one would wind up with an infinite number of subdivisions of $\mathbb{R}^{2}$ (or $\mathbb{R}^{\mathrm{n}}$ ), each subdivision $R(i)$ defined by which subdivision the system would wind up in under which harvest if it began in $R(i)$. Each subdivision would have its own coefficients for the value function because although each would have its coefficient matching equations analogous to (A.1), (A.2) and (A.3), they would all be different from those equations because they would not have the 
unique feature of a universal stockout in bad weather. (Tinkering with these equations suggests that the coefficients converge far from the origin.) Thus we would wind up with a very complicated piecewise quadratic function, over which it would be very hard to optimize ${ }^{3}$.

Thus, proving that the equilibrium of Propositions 2-5 would survive the removal of capacity constraints is an unsolved problem. It is curious: adding the non-negativity constraint robs us of tractability; adding a capacity constraint then gives it back.

\section{Some Notes on the Behaviour of Prices, and Numerical Examples.}

Perhaps the main reason to study this sort of model is to see what implications it might have for the behaviour of primary commodity prices, an area of huge policy interest. As a quick exercise in that line, we here derive price autoregression functions under the different market structures. Deaton and Laroque (1991) studied these functions under perfect competition for a very broad class of demand functions and found that they all were piecewise linear (for i.i.d. harvests), rising at the reciprocal of $\beta(1-\delta)$ up to a threshold price, then becoming flat beyond that price. The former portion corresponded to normal harvests and the latter to drought years. Here we will offer minor revisions.

We will suppress superscripts in what follows; $\Sigma$ s will mean the sum of all speculators' sales, and so on. Suppose one has an equilibrium in which $\sum s=\gamma+(1-\alpha) \sum I$ if $h=h^{+}$and $\sum s$ $=\sum \mathrm{I}$ if $\mathrm{h}=\mathrm{h}^{-}$. Then if $\sum \mathrm{I}=\overline{\mathrm{I}}$ and $\mathrm{h}=\mathrm{h}^{+}, \mathrm{p}=\mathrm{a}-\mathrm{b}\left[\gamma+(1-\alpha) \overline{\mathrm{I}}+\mathrm{h}^{+}\right]$ $=a-b\left[\gamma+h^{+}\right]-b(1-\alpha) \bar{I}$. Thus, in a normal year the maximum possible price is

${ }^{3}$ A special case of this extended model, with $h^{-}=0$ and $\rho=0$, would yield a resource extraction game closely akin to that of Loury (1990), but without any indivisibility. 
$\mathrm{p}_{\max }^{+} \equiv \mathrm{a}-\mathrm{b}\left[\gamma+\mathrm{h}^{+}\right]$. In a drought year, the price is always at least $\mathrm{p}_{\min }^{-} \equiv \mathrm{a}-\mathrm{b}\left[\Sigma \mathrm{I}_{\max }+\mathrm{h}^{-}\right]$

$$
=a-b\left[-\frac{\gamma(1-\delta)}{(1-\alpha(1-\delta))}+h^{-}\right] \text {. }
$$

Thus, drought prices are always higher than normal year prices if $p_{\min }^{-}>p_{\max }^{+}$, which is true if $\left(\mathrm{h}^{+}-\mathrm{h}^{-}+\gamma\right)>\sum \mathrm{I}_{\max }$, which we will assume to be true. In that case, we can divide the autoregression function into two portions, the part above $\mathrm{p}_{\min }^{-}$corresponding to droughts and the rest to normal years.

Pick some price $\overline{\mathrm{p}}$ between $\mathrm{p}_{\max }^{+}$and $\mathrm{p}_{\min }^{-}$. Then given a price $\mathrm{p}<\overline{\mathrm{p}}$ we can deduce that $\sum \mathrm{I}$ $=(1 / b(1-\alpha))\left(a-b\left[\gamma+h^{+}\right]-p\right)$. Then since $\Sigma I_{t+1}=(1-\delta)\left(\alpha \sum I_{t}-\gamma\right)$, the expectation of next year's price is given by:

$$
\begin{aligned}
E_{\tilde{h}}[\tilde{p}] & =\rho\left(a-b\left[\gamma+h^{+}+(1-\alpha)(1-\delta)\left[\frac{\left(a-b\left[\gamma^{+} h^{+}-p\right) \alpha\right.}{b(1-\alpha)} \gamma\right]\right)\right. \\
& +(1-\rho)\left(a-b\left[(1-\delta)\left[\frac{\left(a-b\left[\gamma+h^{+}\right]-p\right) \alpha}{b(1-\alpha)} \gamma\right]+h^{-}\right]\right)
\end{aligned}
$$

(where tildes refer to next period random variables)

$$
\begin{aligned}
=a-b[\rho \gamma+\bar{h} & \left.+(\rho(1-\alpha)+(1-\rho))(1-\delta)\left[\frac{\left(a-b\left[\gamma+h^{+}\right]\right) \alpha}{b(1-\alpha)}-\gamma\right]\right] \\
& +\left(\rho \alpha(1-\delta)+\frac{(1-\rho) \alpha(1-\delta)}{(1-\alpha)}\right) p .
\end{aligned}
$$

Thus the function is linear for $\mathrm{p}<\overline{\mathrm{p}}$. For the competitive equilibrium, this reduces to $E_{\tilde{h}}[\tilde{p}]=k / \beta(1-\delta)+p / \beta(1-\delta)$, as it should. Note as well that $\gamma$ does not enter into the slope; only $\alpha$ does. Therefore, the slope of the autoregression function is independent of the number of speculators in the market and so always equals $1 /(1-\delta) \beta$. The intercept, however, does depend 
on $\gamma$. The competitive intercept is a lower bound, since $E_{\tilde{h}}[\tilde{p}]$ below the competitive autoregression function would mean expected losses on every unit held. Thus, this may be a way of identifying empirically markets whose speculators are protected from entry: if we know somehow that marginal holding costs are small $(\mathrm{k} \approx 0)$, then we could look for a positive intercept for the price autoregression function.

For $\mathrm{p}>\overline{\mathrm{p}}$, we know that next year's stocks will be zero, so

$$
\begin{gathered}
\mathrm{E}_{\tilde{\mathrm{h}}}[\tilde{\mathrm{p}}]=\rho\left(\mathrm{a}-\mathrm{b}\left[\gamma+\mathrm{h}^{+}\right]\right)+(1-\rho)\left(\mathrm{a}-\mathrm{bh}^{+}\right) \\
=\mathrm{a}-\mathrm{b}[\overline{\mathrm{h}}+\rho \gamma] .
\end{gathered}
$$

This is decreasing in $\gamma$; the more competitive speculation is, the more that will be purchased by speculators in the aftermath of a drought (the more negative $\gamma$ will be), and so the higher prices will be after the drought. Thus, more competition means higher price expectations during a drought.

Further, it will mean a less disperse asymptotic price distribution. In every state of nature, high prices (that is, prices above $\bar{p}$ ) will be lower and low prices higher when there is more competition. To see this, note that with the above notation, if at time $t$ there have been $T$ normal harvests since the last drought, inventories will be given by:

$$
\begin{gathered}
\sum I_{t}=-(1-\delta) \gamma+(1-\delta) \alpha \sum I_{t-1} \\
=(1-\delta) \gamma-(1 \delta)^{2} \alpha \gamma+(1-\delta)^{2} \alpha^{2} \sum I_{t-2} \\
=\ldots=-\left(\frac{1-(1-\delta)^{T+1} \alpha^{T+1}}{1-(1-\delta) \alpha}\right)(1-\delta) \gamma
\end{gathered}
$$


This is clearly decreasing in $\gamma$, so an increase in the number of speculators, by lowering $\gamma$, will increase the quantity of stocks held after any harvest sequence and thus increase the amount that will be dumped on the market in drought. Thus, any price above $\bar{p}$ will be lowered. Further, sales in year $t$ if it is a year with a normal harvest are:

$$
\begin{gathered}
\sum s_{t}=\gamma+(1-\alpha) \sum I_{t} \\
=\left[1-(1-\alpha)(1-\delta)\left(\frac{1-(1-\delta)^{T+1} \alpha^{T+1}}{1-(1-\delta) \alpha}\right)\right] \gamma \\
=\left(\frac{\delta+(1-\delta)^{T+1} \alpha^{T+1}}{1-(1-\delta) \alpha}\right) \gamma
\end{gathered}
$$

With more speculators, less will be sold for any given level of stocks carried in, but there will also be more stocks carried in for given harvest sequence. The former effect dominates, as the above expression shows, and a fall in $\gamma$ reduces sales in any year with a normal harvest, thus raising any price below $\bar{p}$. Thus, with more speculators, the price distribution is drawn in toward this intermediate level of $\bar{p}$, and prices are made less volatile.

To give some idea of magnitudes, let us call the "base case" a model with $\rho=3 / 4, h^{+}=60$, $\mathrm{h}^{-}=20, \mathrm{a}=100, \mathrm{~b}=1, \beta=0.97, \mathrm{n}=2, \delta=0.1, \mathrm{k}=1$. In that case, $\alpha=0.7414$, and the intercept of aggregate sales is -3.8181 in the case of perfect competition and -2.5454 for the duopoly. Thus, there is not much storage; maximum aggregate stocks are 10.3269 and 6.8846 respectively, in a market in which every four years on average production drops by 40 units. The intercepts for the price autoregression function are $1.1455(=\mathrm{k} / \beta(1-\delta))$ and 2.1573 , so the increase in the intercept due to market power can be quite significant. With everything else unchanged but $k=0$, the autoregression intercept is still 1.3937. (Would that be far enough away from zero to 
distinguish them econometrically?)

To demonstrate how easy it is to satisfy $(3.2)(\mathrm{h})$ in the base case, $\mathrm{k}$ here equals 34.8501 . We need the individual stock maximum to be less than $k /(n+1)$, but here the individual maximum equals 3.4423

Behaviour and profits are fairly sensitive to cost parameters. If we bring holding costs down from one per unit to zero, the maximum aggregate inventories go up by about half, to 14.2239 and 9.4826, and individual profits just coming out of a drought double: $\mathrm{e}_{0}$ goes from 109.2371 to 207.2355. However, it is surprisingly easy to erase those gains by letting a few more speculators into the market. With $\mathrm{k}=0$ and $\mathrm{n}=20$, $\mathrm{e}_{0}$ drops to 4.2293 ! Whatever available weapons for entry deterrence would surely be used. Importantly, we see that a fairly small fixed cost will be enough to keep entry down to a small number of players. This is apparent from Proposition 5, which shows that profits fall with entry roughly at the rate $\mathrm{n}^{2}$.

An interesting experiment is to vary the popularity of the good alone, that is, the parameter a. Paradoxically, when the good becomes too popular the speculators can practically be put out of business. Returning to the base case and setting $a=120$, we find that maximum aggregate stocks fall to 0.4287 for competition and 0.2858 for the duopoly. $\mathrm{e}_{0}$ takes a value of 0.1882 . Why this malaise among traders of what is clearly a hot product? The parallel shift of the demand schedule up 20 dollars increases the buying price by 20 dollars ceteris paribus, but the discounted selling price by only $\beta(1-\delta) 20 \approx 17$ for a unit to be sold next year and less for units sold later. Thus, in present value, the speculator is out at least 3 dollars per unit per period. By the same token, consumer weariness of the good which sends the parameter a down to 80 is a gift to the speculators. Maximum aggregate stocks increase to 20.2252 and 13.4384 respectively, 
and $\mathrm{e}_{0}$ shoots up to 418.9990 .

Another way of viewing this is as the effect of a subsidy to consumers of the good. Such a subsidy reduces storage and makes the price more volatile, clearly exacerbating the welfare losses resulting from oligopoly. Perhaps this can help explain the almost ubiquitous tendency toward public sector storage accompanying grain subsidies in the post-war era. A last interpretation, if the producers and consumers lie on opposite sides of a border, is as the effect of a tariff. A reduction in a could be brought about by a rise in the consumer country's (specific) tariff; paradoxically, the traders would favor the tariff against their produce (perhaps unless it was raised at a moment when they already held large stocks). On the other hand, a rise in b would represent an increase in a variable import levy, a tariff linearly falling in $p_{t}$. This would hurt the traders if $b$ was initially low and help them if it was large (for example, when $b=0.5, e_{0}$ $=949.6697$; when $b=1, e_{0}=109.2371$; and when $b=1.1, e_{0}=259.6495 . e_{0}$ follows a parabolic-type path in $b$ with minimum around $b=0.8$ ).

The strident opposition of the international grain oligopoly to the Common Agricultural Policy is well documented (Morgan, 1979, pp. 130-4). Perhaps if the EEC had imposed a specific tariff rather than a variable levy it would have found a different response?

(3.2)(h) is easily satisfied in all of these examples. Thus, the model does work over a wide range of parameter values and offers a rich variety of comparative dynamics.

\section{Conclusion.}

We have presented a model of commodity trading and speculation in the presence of 
restricted entry into speculation. We have solved the model for an infinite horizon subgame perfect equilibrium which turns out to be extremely tractable, and has three properties worth pointing out: One, the independence property: in equilibrium, no speculator's behaviour affects the others'. Two, a persistent movement toward equal levels of stocks regardless of their initial distribution. Finally, profits fall at the rate $n^{2}$ (Proposition 5) while the difference in aggregate behaviour from perfect competition falls at the rate $\mathrm{n}$ (Propositon 4); therefore even a small fixed cost of entry would keep the market concentrated and noticeably different from a competitive one.

Changes in the number of firms result in parallel shifts of equilbrium behaviour functions. These have an effect on price dispersion, which falls as the number of traders rises. A surprising result is that the oligopolists would lobby in favor of a tariff against their produce, and that this tariff would improve welfare by increasing storage.

The verisimilitude of the model is in doubt, especially regarding property two, but perhaps it is a start and perhaps useful as a benchmark. A natural next step would be to search out other equilibria and to find out which properties depend on straight-line demand functions and which are robust. 


\section{Appendix: Proof of Proposition 1.}

Proof: Consider speculator 1. In a year with a normal harvest, she will maximize:

$$
\begin{gathered}
s^{1}\left(a-b\left[\sum_{j=1}^{n} s^{j}+h^{+}\right]\right)-k\left(I^{1}-s^{1}\right)+\beta E_{\tilde{h}}\left[V^{j}((1-\delta)(I-s), \tilde{h})\right] \\
=s^{1}\left(a-b\left[\sum_{j=1}^{n} s^{j}+h^{+}\right]\right)-k\left(I^{1}-s^{l}\right)+\beta\left[e_{o}+e_{1}(1-\delta)\left(I^{1}-s^{1}\right)+e_{2}(1-\delta)^{2}\left(I^{1}-s^{1}\right) \sum_{j=1}^{n}\left(I^{j}-s^{j}\right)\right] .
\end{gathered}
$$

(We have used the difference equation $I_{t+1}=(1-\delta)\left(I_{t}-s_{t}\right)$.) Taking the derivative with respect to $\mathrm{s}^{1}$ and setting it equal to zero yields:

$$
\begin{gathered}
\mathrm{s}^{1}=\left(2 \mathrm{~b}-2 \beta(1-\delta)^{2} \mathrm{e}_{2}\right)^{-1}\left[\mathrm{a}-\mathrm{b} \mathrm{h}^{+}+\mathrm{k}-\beta(1-\delta) \mathrm{e}_{1}-2 \beta(1-\delta)^{2} \mathrm{e}_{2} \mathrm{I}^{1}-\beta(1-\delta)^{2} \mathrm{e}_{2} \sum_{j=1}^{\mathrm{n}} \mathrm{I}^{\mathrm{j}}-\mathrm{b}-\beta(1-\delta)^{2} \mathrm{e}_{2} \sum_{\mathrm{j} \neq 1} \mathrm{~s}^{\mathrm{j}}\right] \\
=\mathrm{f}-\mathrm{g}\left(\mathrm{I}^{1}+\sum_{\mathrm{j}=1}^{\mathrm{n}} \mathrm{I}^{\mathrm{j}}\right)-(1 / 2) \sum_{\mathrm{j} \neq 1} \mathrm{~s}^{\mathrm{j}}
\end{gathered}
$$

But since this is true for all speculators simultaneously,

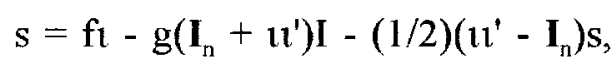

where $\mathbf{t}$ is a vector of ones and $\mathbf{I}_{\mathrm{n}}$ is the identity matrix. This implies:

$$
\mathrm{s}=2 \mathrm{f} /(\mathrm{n}+1) \mathrm{t}-2 \mathrm{gI}
$$

using the fact that $\left[\mathbf{I}_{n}-(1 /(n+1)) \mathfrak{u}\right]\left[\mathbf{I}_{n}+\imath^{\prime}\right]=\mathbf{I}_{n}$. Now, noting from the definition of $g$ that it is negative (since $e_{2}$ is assumed negative) and less than $1 / 2$ in absolute value, it is clear that the difference equation $I_{t+1}=(1-\delta)\left(I_{t}-s_{t}\right)=-2(1-\delta) f /(n+1) t+(1-\delta)(1+2 g) I_{t}$ for normal years is stable, and that the maximum level any speculator's inventories could reach after an infinite run of good harvests is

$$
I_{\max }=-\frac{2(1-\delta) f}{(1-(1+2 g)(1-\delta))(n+1)}
$$

Thus, by (3.2)(h), none of the speculators will ever exceed capacity, so condition $(3.1)(\mathrm{c})$ is 
satisfied. $(3.1)(b)$ is also trivially satisfied as long as $\sum_{j=1}^{n} K^{j}$ is less than $h^{+}$. Further, since by assumption $f<0$, inventories are never negative and $(3.1)(a)$ is satisfied. (Note that the equilibrium sales function has a negative intercept. This means that in the first good year after a drought, speculators buy.)

Condition $(3.1)(f)$ is satisfied by the stability of the difference equation if we define $D$ as $\left\{\mathrm{I}: \mathrm{I}^{\mathrm{j}} \in\left[0, \mathrm{~K}^{\mathrm{j}}\right] \forall \mathrm{j}\right\}$. Condition $(3.1)(\mathrm{d})$ is satisfied for $\mathrm{h}=\mathrm{h}^{+}$by construction. (Note that $\mathrm{e}^{2}<0$ guarantees that the second order condition is satisfied.) For $h=h^{-}$, we must ask under what conditions with the given next-period value function it would be a Nash equilibrium for all parties to stock out. Returning to speculator l's problem discussed above, if there is now a drought on and all others are selling their entire stocks, the level of sales which would satisfy her first order condition is:

$$
\begin{gathered}
\mathrm{s}^{1}=\mathrm{f}-\mathrm{g}\left(\mathrm{I}^{1}+\sum_{\mathrm{j}=1}^{\mathrm{n}} \mathrm{I}^{\mathrm{j}}\right)-(1 / 2) \sum_{\mathrm{j} \neq 1} \mathrm{I}^{\mathrm{j}}=\mathrm{f}-2 \mathrm{gI} \mathrm{I}^{1}-((1+2 \mathrm{~g}) / 2) \sum_{\mathrm{j} \neq 1} \mathrm{I}^{\mathrm{j}} \\
\geq \mathrm{I}^{1} \text { iff } \\
\left(2 \boldsymbol{I}^{\mathbf{1}}+\sum_{j \neq \mathbf{1}} \boldsymbol{I}^{j}\right) \leq \frac{\mathbf{2 f ^ { - }}}{(\mathbf{1}+\mathbf{2 g})}=\frac{\boldsymbol{a}-\boldsymbol{b} \boldsymbol{h}^{-}+\boldsymbol{k}+\boldsymbol{k}-\boldsymbol{\beta}(\mathbf{1}-\boldsymbol{\delta}) \boldsymbol{e}_{\mathbf{1}}}{\boldsymbol{b}} .
\end{gathered}
$$

Therefore, since by $(3.2)(\mathrm{h})$ and our results on the stability of the system,

$$
I^{j} \leq \frac{a-b h^{-}+k-\boldsymbol{\beta}(1-\delta) e_{1}}{b(n+1)} \forall j
$$

at all times, we have established that stockouts are indeed an equilibrium during a drought.

The last condition is the most tedious to check, but also the most informative since it gives us a way of calculating the coefficients. This is $(3.1)(\mathrm{e})$, the recursive condition. Consider the 
case of a normal harvest. We first plug the behavioural functions we have derived into (3.1)(e). We find:

$$
\begin{aligned}
V^{1}\left(I, h^{+}\right)=( & \left.2 f /(n+1)-2 g I^{1}\right)\left(a-b\left[2 n f /(n+1)-2 g \sum_{j=1}^{n} I^{j}+h^{+}\right]\right) \\
& -k\left(I^{1}-2 f /(n+1)+2 g I^{1}\right)+\beta e_{0}+\beta e_{1}(1-\delta)\left(I^{1}-2 f /(n+1)+2 g I^{l}\right) \\
& +\beta e_{2}(1-\delta)^{2}\left(I^{1}-2 f /(n+1)+2 g I^{1}\right)\left(\sum_{j-1}^{n}(1+2 g) I^{j}-2 f /(n+1)\right)
\end{aligned}
$$

The right hand side expression can be gathered into four terms: a constant, a term in $\mathrm{I}^{1}$, a term in $I^{1} \sum_{j=1}^{n} I^{j}$, and a term in $\sum_{j-1}^{n} I^{j}$. The latter has a coefficient equal to:

$2 \mathrm{f} /(\mathrm{n}+1)\left[2 \mathrm{bg}-\beta \mathrm{e}_{2}(1-\delta)^{2}(1+2 \mathrm{~g})\right]=0$, (using $\left.(3.2)(\mathrm{b})\right)$ as required, since the proposed value function has no term linear in $\sum_{j=1}^{n} I^{j}$. Matching up coefficients for $\mathrm{I}^{\mathrm{l}} \sum_{\mathrm{j}=1}^{\mathrm{n}} \mathrm{I}^{\mathrm{j}}$ gives:

$$
\mathrm{e}_{2}^{+}=\beta \mathrm{e}_{2}(1-\delta)^{2}(1+2 \mathrm{~g})^{2}-4 \mathrm{~g}^{2} \mathrm{~b}
$$

Using $e_{2}=\rho e_{2}^{+}+(1-\rho) e_{2}^{-}$and (3.2)(b) to eliminate the g's turns this into the cubic equation at the beginning of the statement of this proposition, and since $e_{2}$ was defined as a root of that equation, (A.1) is satisfied. Matching coefficients of $I^{1}$ yields:

$$
\begin{aligned}
& e_{1}^{+}=-2 g\left(a-b\left[2 n f /(n+1)+h^{+}\right]\right)-k(1+2 g)+\beta e_{1}(1-\delta)(1+2 g) \\
& -\beta e_{2}(1-\delta)^{2}(1+2 g) 2 n f /(n+1) \\
& =-2 g\left(a-b h^{+}\right)-k(1+2 g)+\beta e_{1}(1-\delta)(1+2 g)(u \operatorname{sing}(3.2)(b))
\end{aligned}
$$

Combining this with $(3.2)(\mathrm{d})$ shows that it is identical to the first statement of (3.2)(c). Thus, (A.2) holds. Finally, matching up constant terms gives us:

$$
\begin{aligned}
e_{0}^{+}=2 f /(n+1)(a-b[2 n f /(n+1) & +h])+\beta e_{0}-\beta e_{1}(1-\delta)^{2} f /(n+1)+\beta e_{2}(1-\delta)^{2} 4 f^{2} n /(n+1)^{2} \\
& +2 k f /(n+1)
\end{aligned}
$$

Using (3.2)(g) to eliminate the $\mathrm{e}_{0}^{+}$and bringing the $\mathrm{e}_{0}$ to the left hand side shows that (A.3) is equivalent to the definition of $e_{0}$, which is the first statement of (3.2)(f). Thus (A.3) holds. 
It is trivial to check the coefficients for the drought case. This is, in fact, done in the note in the statement of the proposition. We conclude that $(3.1)($ e) holds, as required. Q.E.D. 


\section{Bibliography}

Bauer, P.T. (1953). "Concentration in Tropical Trade: Some Aspects and Implications of Oligopoly," Economica, November 1953, pp. 302-21. Reprinted in P.T. Bauer and B.S. Yamey, Markets, Market Control and Marketing Reform, Weidenfeld and Nicolson, Iontn, 1968.

Deaton, Angus and Guy Laroque (1991). "On the Behavior of Commodity Prices." Forthcoming, Review of Economic Studies.

Hartzmark, Micheal L. (1987). "Returns to Individual Traders of Futures: Aggregate Results," Journal of Political Economy, 95:6, pp. 1292-1306.

Houthakker, H.S. (1957). "Can Speculators Forecast Prices?" Review of Economics and Statistics, 39:2, pp. 143-51.

Judd, Kenneth (1990). "Cournot versus Bertrand: A Dynamic Resolution." Typescript.

Kirman, Alan P. and Matthew J. Sobel (1974). "Dynamic Oligopoly with Inventories". Econometrica, Vol. 42, No.2.

Kydland, Finn (1975). "Noncooperative and Dominant Player Solutions in Discrete Dynamic Games." International Economic Review, Vol. 16, No. 2, pp. 321-35.

Loury, Glenn C (1990). "Tacit Collusion in a Dynamic Duopoly with Indivisible Production and Cumulative Capacity Constraints." Harvard University, mimeo.

Maizels, Alfred (1984). "A Conceptual Framework for Analysis of Primary Commodity Markets", World Development, 12:1, pp. 25-42.

Morgan, Dan (1979). Merchants of Grain. The Viking Press, New York.

Newbery, David M (1984). "Commodity Price Stabilization in Imperfect or Cartelized Markets." Econometrica, 52:3, pp. 563-78.

Newbery, David M. and Joseph E. Stiglitz (1981). The Theory of Commodity Price Stabilization: A Study in the Economics of Risk. 1981: Clarendon Press, Oxford.

Reinganum, Jennifer F. and Nancy L. Stokey (1985). "Oligopoly Extraction of a Common Property Natural Resource: The Importance of the Period of Commitment in Dynamic Games." International Economic Review, 26:1, pp. 161-73.

Sargent, Thomas (1987). Dynamic Macroeconomic Theory. Harvard University Press, Cambridge. 
Scheinkman, J.A., and J. Schechtman, (1983). "A Simple Competitive Model with Production and Storage," Review of Economic Studies, 50, pp. 427-41.

Shapiro, Carl (1988). "Theories of Oligopoly Behaviour". In Handbook of Industrial Economics, Robert D Willig and Richard Shmalensee, ed., Amsterdam, North Holland.

Sundaram, Rangarajan K. (1989). "Perfect Equilibrium in Non-randomized Strategies in a Class of Symmetric Dynamic Games," Journal of Economic Theory, 47, pp. 153-77.

Sutton, John (1991). Sunk Costs and Market Structure: Price Competition, Advertising, and The Evolution of Concentration. Cambridge, Massachusetts: The MIT Press.

Williams, Jeffrey and Brian Wright (1991). Storage and Commodity Markets, Cambridge: Cambridge University Press. 


\section{TABLE I: Notation}

(3.2)(a)

$$
e_{2}^{+}=\left(e_{2}+(1-\rho) b\right) / \rho ; \quad e_{2}^{-}=-b
$$

(3.2)(b)

$$
g=\frac{\beta(1-\delta)^{2} e_{2}}{2\left(b-\beta(1-\delta)^{2} e_{2}\right)}
$$

(3.2)(c)

$$
e_{1}=(1-\beta \rho(1-\delta)(1+2 g))^{-1}\left[(a-b \bar{h})-(1+2 g) \rho\left(a-b h^{+}+k\right)\right] ;
$$

$$
e_{1}^{+}=\left(e_{1}-(1-\rho)\left(a-b h^{-}\right)\right) / \rho ; \quad e_{1}^{-}=a-b h^{-} ;
$$

$$
f=\frac{a-b h^{+}+k-\beta(1-\delta) e_{1}}{2\left(b-\beta(1-\delta)^{2} e_{2}\right)}
$$

similarly $\mathrm{f}^{-}$has $\mathrm{h}^{-}$instead of $\mathrm{h}^{+}$;

(3.2)(f)

$$
e_{0}=\frac{2 f}{(n+1)} \frac{\rho}{(1-\beta)}\left\{a-b\left(\frac{2 n f}{(n+1)}+h^{+}\right)+k-\beta e_{1}(1-\delta)+\beta e_{2}(1-\delta)^{2} \frac{2 n f}{(n+1)}\right\} ; \text { and }
$$

$(3.2)(\mathrm{g})$

$$
e_{0}^{+}=\frac{(1-(1-\rho) \beta) e_{0}}{\rho} ; e_{0}^{-}=\beta e_{0} \text {. }
$$




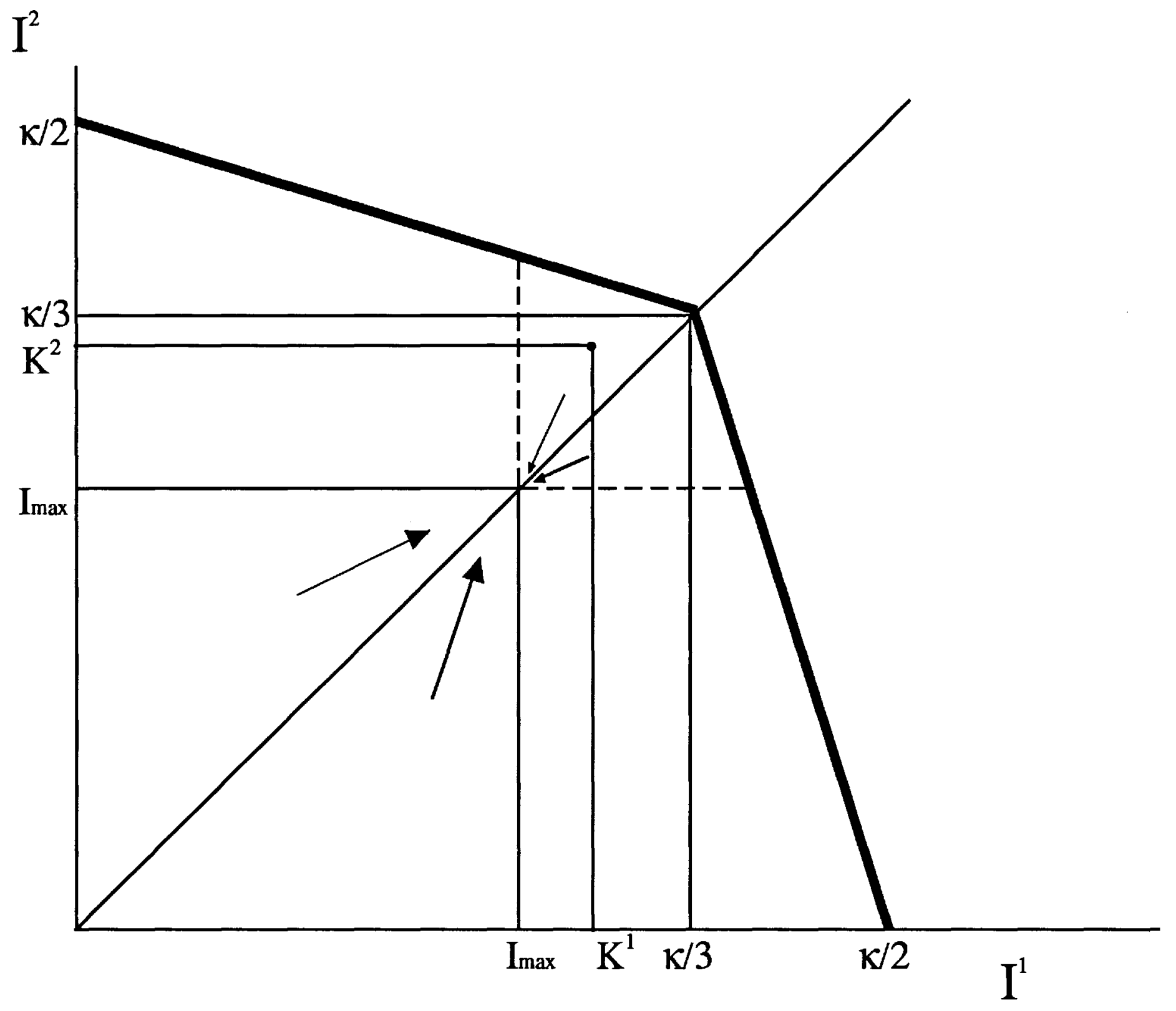

Figure 1.1(a):

Normal Year 


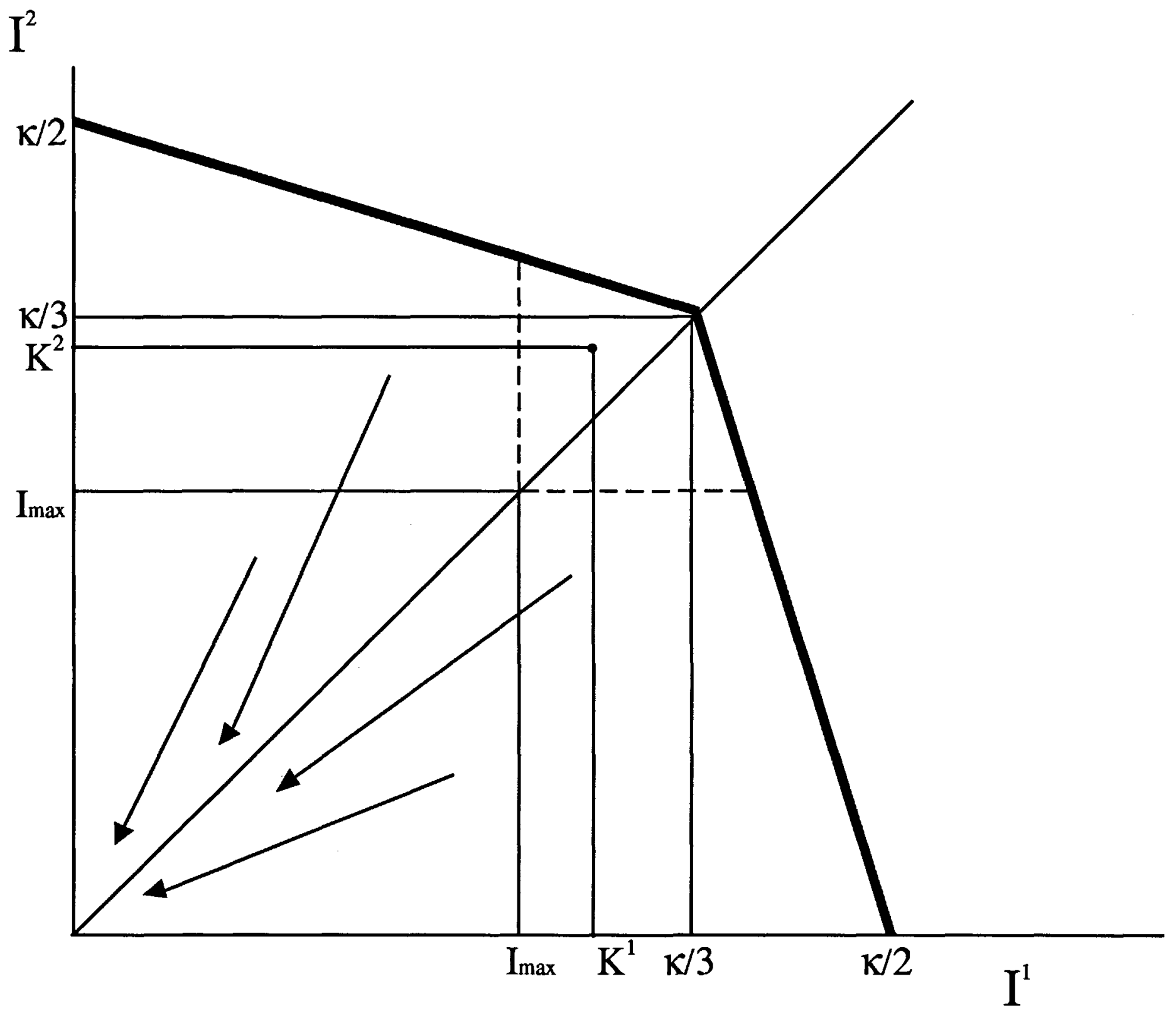

Figure 1.1(b):

Drought 